\title{
Two Improved Homomorphic Techniques Used in Functional Encryption Schemes
}

\author{
Chengbo $\mathrm{Xu}^{1}$, Shuying Yang ${ }^{2}$ \\ ${ }^{1}$ School of Mathematical Sciences, University of Jinan, Jinan 250022, \\ Shandong Province, P. R. China \\ ${ }^{2}$ Department of Data and Computer Science, Shandong Women's University, Jinan 250300, \\ Shandong Province, P. R. China
}

\begin{abstract}
In this paper, we analyze the key homomorphic technique used in constructions of functional encryption schemes and point out its weakness in efficiency. Based on this, we propose two improved homomorphic techniques and show their advantages and weaknesses through the method of comparison.
\end{abstract}

\section{Introduction}

With the development of cloud computing and other complex networks, important progress has been witnessed recently in the field of computing on encrypted data. A number of concepts and constructions of cryptographic primitives have been raised, such as Attribute Based Encryption (Gorbunov et al. 2013; Boneh et al. 2014), Fully Homomorphic Encryption (Gentry 2009), Functional Encryption (Boneh et al. 2011; Gary et al. 2013; Agrawal 2017).

Among them, functional encryption was formalized by Boneh et al. (2011) to unify and extend the notions of identity based encryption, attribute-based encryption and Predicate Encryption. Since then, considerable progress has been made towards constructing functional encryption scheme. In 2014, Boneh et al. (2014) proposed an attributed based encryption scheme for general circuits. Based on this, Gorbunov et al. (2015) designed a predicate encryption. However, in the construction, there are two sources of leakage that prevent the scheme from achieving the strong attributehiding guarantee (Agrawal 2017). In order to conquer these draw-backs, Brakerski et al. (2017) proposed an improved predicate encryption scheme.

In this paper, we abstract the key homomorphic technique used in the construction of this series of functional encryption scheme. Based on this, we propose two improved homomorphic techniques and show their advantages and weaknesses through the method of comparison.

\section{Basic homomorphic Techniques}

In this section, we first review BGGHNSVV attributebased encryption scheme briefly (Boneh et al. 2014), and then analyze the basic homomorphic techniques used in its construction.

\subsection{Review Of BGGHNSVV Scheme}

Let $n$ be an integer, and $q=q(n), \quad m=\Theta(n \log q)$. Let $G \in \mathbb{Z}_{q}^{n \times m}$ be the gadget matrix. We review the attributebased encryption scheme as follows:

- $\operatorname{Setup}\left(1^{\lambda}, l\right)$ : Sample a matrix with associated trapdoor $\left(A, T_{A}\right) \leftarrow \operatorname{TrapGen}\left(1^{m}, 1^{n}, q\right)$; Choose random matrices $A_{i} \in \mathbb{Z}_{q}^{n \times m}$ for $i=1, \cdots, l$, and $D \in \mathbb{Z}_{q}^{n \times m}$. Set $m p k=\left(A, D, A_{1}, \cdots, A_{l}\right)$, and $m s k=\left(m p k, T_{A}\right)$.

- $\operatorname{Enc}\left(m p k, x \in \mathbb{Z}_{q}^{l}, \mu \in\{0,1\}^{m}\right)$ : Choose a secret vector $s \leftarrow \mathbb{Z}_{q}^{n}$ and error terms $e_{o}, e_{1} \leftarrow \chi^{m}$, and set $H=\left(A\left|x_{1} G+A_{1}\right| \cdots \mid \quad x_{l} G+A_{l}\right) \in \mathbb{Z}_{q}^{n \times(l+1) m}$, $e=\left(\begin{array}{lllll}I_{m}\left|S_{1}\right| & \cdots & S_{l}\end{array}\right)^{T} \quad e_{0} \quad \in \mathbb{Z}_{q}^{(l+1) m}$. Set $c=\left(H^{T} s+e, D^{T} s+e_{1}+\lceil q / 2\rceil \mu\right) \in \mathbb{Z}_{q}^{(l+2) m}$.

- $\operatorname{KeyGen}(m s k, f)$ : Compute $A_{f}=\operatorname{Eval}_{\mathrm{pk}}\left(f,\left(A_{1}\right.\right.$, $\left.\cdots, A_{l}\right)$ ); Run the algorithm SampleRight $\left(A, T_{A}\right.$, $\left.A_{f}, D, \sigma\right)$ to generate low-norm matrix $R_{f}$ such that $\left(A \mid A_{f}\right) \cdot R_{f}=D$. Output $s k_{f}:=R_{f}$.

- $\operatorname{Dec}\left(s k_{f},(x, c)\right)$ : If $f(x) \neq 0$, output $\perp$. Otherwise, parse the ciphertext as $c=\left(c_{i n}\right.$, 
$\left.c_{1}, \cdots, c_{l}, c_{\text {out }}\right) \in \mathbb{Z}_{q}^{(l+2) m}$, and compute $c_{f}=\mathrm{Eval}_{\mathrm{ct}}$

$\left(f,\left\{\left(x_{i}, B_{i}, c_{i}\right)\right\}_{i=1}^{l}\right) \in \mathbb{Z}_{q}^{m}$. Output $\operatorname{Round}\left(c_{\text {out }}-R_{f}^{T}\left(c_{\text {in }} \mid c_{f}\right)\right) \in\{0,1\}^{m}$.

\subsection{Basic Homomorphic Techniques}

In this subsection, we abstract the homomorphic techniques used in construction of the BGGHNSVV attribute-based encryption scheme. In literature, the technique is as one of basic tools to make the fine-grain access to the encrypted data feasible in the field of functional encryption.

For convenience, we define some notions as follows: Set parameters $n, q, m$ and $G$ as above. Choose uniform randomly matrices $B_{1}, B_{2}, \cdots, B_{t} \in \mathbb{Z}_{q}^{n \times m}$, and values $x_{1}, x_{2}, \cdots, x_{t} \in \mathbb{Z}_{q}$. Compute $c_{i}=B_{i}+x_{i} G$ for $i=1,2, \cdots, t$.

\subsubsection{Homomorphic Technique For Weighted Addition Gates}

For a weighted addition gate $f: \mathbb{Z}_{q}^{t} \rightarrow \mathbb{Z}_{q}$,

$$
f(x)=\alpha_{1} x_{1}+\alpha_{2} x_{2}+\cdots+\alpha_{t} x_{t}
$$

Do the following computation:

$$
\begin{aligned}
B_{f}= & E_{v a l}\left(f, B_{1}, \cdots, B_{t}\right) \\
= & A_{1} G^{-1}\left(\alpha_{1} G\right)+\cdots+A_{t} G^{-1}\left(\alpha_{t} G\right) \\
c_{f}= & E_{v a l}^{c t}\left(f, B_{1}, \cdots, B_{t}, c_{1}, \cdots, c_{t}, x_{1}, \cdots, x_{t}\right) \\
= & c_{1} G^{-1}\left(\alpha_{1} G\right)+\cdots+c_{t} G^{-1}\left(\alpha_{t} G\right) \\
= & {\left[A_{1} G^{-1}\left(\alpha_{1} G\right)+\cdots+A_{t} G^{-1}\left(\alpha_{t} G\right)\right] } \\
& +\left(\alpha_{1} x_{1}+\cdots \alpha_{t} x_{t}\right) G \\
= & B_{f}+\left(\alpha_{1} x_{1}+\cdots \alpha_{t} x_{t}\right) G \\
= & B_{f}+f\left(x_{1}, \cdots, x_{t}\right) G
\end{aligned}
$$

This type of representation is from Boneh et al. (2014). In order to describe the basic homomorphic more clearly, we make a change of the presentation as follows:

$$
\begin{aligned}
H_{f} & =\operatorname{EvalF}\left(f, B_{1}, \cdots, B_{t}\right) \\
& =\left[G^{-1}\left(\alpha_{1} G\right), \cdots, G^{-1}\left(\alpha_{t} G\right)\right]^{T}
\end{aligned}
$$

Thus, we have

$$
\begin{aligned}
B_{f}= & A_{1} G^{-1}\left(\alpha_{1} G\right)+\cdots+A_{t} G^{-1}\left(\alpha_{t} G\right) \\
= & \left(A_{1}, \cdots, A_{t}\right)\left(G^{-1}\left(\alpha_{1} G\right)^{T} \quad \cdots \quad G^{-1}\left(\alpha_{t} G\right)^{T}\right)^{T} \\
= & \left(A_{1}, \cdots, A_{t}\right) H_{f} \\
H_{f, x} & =\operatorname{EvalFX}\left(f, B_{1}, \cdots, B_{t}, c_{1}, \cdots, c_{t}, x_{1}, \cdots, x_{t}\right) \\
& =\left[G^{-1}\left(\alpha_{1} G\right), \cdots, G^{-1}\left(\alpha_{t} G\right)\right]^{T}
\end{aligned}
$$

Similarly, we have the following formula:

$$
\begin{aligned}
c_{f} & =c_{1} G^{-1}\left(\alpha_{1} G\right)+\cdots+c_{t} G^{-1}\left(\alpha_{t} G\right) \\
& =\left(c_{1}, \cdots, c_{t}\right)\left(G^{-1}\left(\alpha_{1} G\right)^{T} \quad \cdots \quad G^{-1}\left(\alpha_{t} G\right)^{T}\right)^{T} \\
& =\left(c_{1}, \cdots, c_{t}\right) H_{f, x}
\end{aligned}
$$

From above formulations, we may realize that the matrices $H_{f}$ and $H_{f, x}$ play a vital role of homomorphically transforming the original resource $B_{1}$,
$B_{2}, \cdots, B_{t}$ and $c_{1}, c_{2}, \cdots, c_{t}$ to $B_{f}$ and $c_{f}$, respectively. Therefore, the matrices $H_{f}$ and $H_{f, x}$ represent the basic homomorphic technique for weighted addition gates.

\subsubsection{Homomorphic Technique for Multi-plication Gates}

For a weighted multiplication gate $f: \mathbb{Z}_{q}^{t} \rightarrow \mathbb{Z}_{q}$,

$$
f(x)=\alpha_{1} x_{1} x_{2} \cdots x_{t}
$$

Since process of this homomorphic technique is complex for general $t$, we set $t=3$ here to illustrate the technical detail of computational process as follows:

$$
\begin{gathered}
c_{1}\left(x_{2} x_{3}\right) G^{-1}(\alpha G)=\left(x_{2} x_{3}\right) B_{1} G^{-1}(\alpha G)+\left(\alpha x_{1} x_{2} x_{3}\right) G \\
c_{2}\left(x_{3}\right) G^{-1}\left(-B_{1} G^{-1}(\alpha G)\right) \\
=x_{3} B_{2} G^{-1}\left(-B_{1} G^{-1}(\alpha G)\right)-\left(\alpha x_{2} x_{3}\right) B_{1} \\
c_{3} G^{-1}\left(-B_{2} G^{-1}\left(-B_{1} G^{-1}(\alpha G)\right)\right) \\
=B_{3} G^{-1}\left(-B_{2} G^{-1}\left(-B_{1} G^{-1}(\alpha G)\right)\right) \\
-x_{3} B_{2} G^{-1}\left(-B_{1} G^{-1}(\alpha G)\right)
\end{gathered}
$$

Summing above three equations, we obtain

$$
\begin{aligned}
c_{f}= & c_{1} x_{2} x_{3} G^{-1}(\alpha G)+c_{2} x_{3} G^{-1}\left(-B_{1} G^{-1}(\alpha G)\right) \\
& +c_{3} G^{-1}\left(-B_{2} G^{-1}\left(-B_{1} G^{-1}(\alpha G)\right)\right) \\
= & B_{3} G^{-1}\left(-B_{2} G^{-1}\left(-B_{1} G^{-1}(\alpha G)\right)\right) \\
& +\left(\alpha x_{1} x_{2} x_{t}\right) G \\
= & B_{f}+\left(\alpha x_{1} \cdots x_{t}\right) G
\end{aligned}
$$

From the equation, we are able to obtain

$$
\begin{aligned}
& H_{f}=\operatorname{EvalF}\left(f, B_{1}, B_{2}, B_{3}\right) \\
& =\left(\begin{array}{c}
\alpha x_{2} x_{3} E \\
\alpha x_{3} G^{-1}\left(-B_{1} G^{-1}(\alpha G)\right) \\
G^{-1}\left(-B_{2} G^{-1}\left(-B_{1} G^{-1}(\alpha G)\right)\right)
\end{array}\right) \\
& H_{f, x}=\operatorname{EvalFX}\left(f, B_{1}, B_{2}, B_{3}, c_{1}, c_{2}, c_{3}, x_{1}, x_{2}, x_{3}\right) \\
& =\left(\begin{array}{c}
O \\
O \\
G^{-1}\left(-B_{2} G^{-1}\left(-B_{1} G^{-1}(\alpha G)\right)\right)
\end{array}\right)
\end{aligned}
$$

For general $t$, the outputs of algorithm $\operatorname{EvalF}(\cdot)$ and EvalFX $(\cdot)$ should be

$$
\begin{aligned}
& H_{f}=\operatorname{EvalF}\left(f, B_{1}, \cdots, B_{t}\right) \\
& =\left(\begin{array}{c}
\left(x_{2} \cdots x_{t}\right) G^{-1}(\alpha G) \\
\left(x_{3} \cdots x_{t}\right) G^{-1}\left(-B_{1} G^{-1}(\alpha G)\right) \\
\vdots \\
G^{-1}\left(-B_{t-1} G^{-1}\left(-B_{t-2} G^{-1}\left(\cdots G^{-1}\left(-B_{1} G^{-1}(\alpha G)\right)\right)\right)\right)
\end{array}\right) \\
& H_{f, x}=\operatorname{EvalFX}\left(f, B_{1}, \cdots, B_{t}, c_{1}, \cdots, c_{t}, x_{1}, \cdots, x_{t}\right) \\
& =\left(\begin{array}{c}
O \\
O \\
G^{-1}\left(-B_{t-1} G^{-1}\left(-B_{t-2} G^{-1}\left(\cdots G^{-1}\left(-B_{1} G^{-1}(\alpha G)\right)\right)\right)\right)
\end{array}\right)
\end{aligned}
$$

\subsubsection{Homomorphic Technique for General Arithmetic Circuits}


For general arithmetic circuits $f: \mathbb{Z}_{q}^{t} \rightarrow \mathbb{Z}_{q}$, the input wires are indexed 1 to $t$, the internal wires have indices $t+1, t+2, \cdots,|f|-1$ and the output wire has index $|f|$, which also denotes the size of the circuit. Each gate $g_{\omega}: \mathbb{Z}_{q}^{k_{\omega}} \rightarrow \mathbb{Z}_{q}$ is indexed as a tuple $\left(\omega_{1}, \cdots, \omega_{k_{\omega}}, \omega\right)$ where $k_{\omega}$ is the fan-in of the gate.

By the algorithms for single gates, which compute weighted additions and multiplications as described above, we can conduct the required matrices $H_{f}$ and $H_{f, x}$ inductively input to output gate-by-gate.

\section{Proposed homomorphic techniques}

In this section, we proposed two new homomorphic methods. In both of them, the way to deal with cases of addition gate and general circuits is partially same to corresponding processes of the basic homo-morphic techniques described in section 2. For simplicity, we only point out the different part in our proposed techniques, that is, how to evaluate multiplication gate.

\subsection{Proposed Technique I}

For a weighted multiplication gate $f: \mathbb{Z}_{q}^{t} \rightarrow \mathbb{Z}_{q}$,

$$
f(x)=\alpha_{1} x_{1} x_{2} \cdots x_{t}
$$

We compute matrices $H_{f}$ and $H_{f, x}$ as follows:

$$
\begin{aligned}
& H_{f}=\operatorname{EvalF}\left(f, B_{1}, \cdots, B_{t}\right) \\
&=\left(\begin{array}{c}
\left(\alpha x_{2} \cdots x_{t}\right) E \\
\left(\alpha x_{3} \cdots x_{t}\right) G^{-1}\left(-B_{1}\right) \\
\vdots \\
\alpha G^{-1}\left(-B_{t-1} G^{-1}\left(-B_{t-2} G^{-1}\left(\cdots G^{-1}\left(-B_{1}\right)\right)\right)\right)
\end{array}\right) \\
& H_{f, x}=\operatorname{EvalFX}\left(f, B_{1}, \cdots, B_{t}, c_{1}, \cdots, c_{t}, x_{1}, \cdots, x_{t}\right) \\
& O \\
& O \\
&=\left(\begin{array}{c} 
\\
\alpha G^{-1}\left(-B_{t-1} G^{-1}\left(-B_{t-2} G^{-1}\left(\cdots G^{-1}\left(-B_{1}\right)\right)\right)\right)
\end{array}\right)
\end{aligned}
$$

\subsection{Proposed Technique II}

For a weighted multiplication gate $f: \mathbb{Z}_{q}^{t} \rightarrow \mathbb{Z}_{q}$,

$$
f(x)=\alpha_{1} x_{1} x_{2} \cdots x_{t}
$$

We compute matrices $H_{f}$ and $H_{f, x}$ as follows:

$$
\begin{aligned}
H_{f}= & \operatorname{EvalF}\left(f, B_{1}, \cdots, B_{t}\right) \\
= & \left(\begin{array}{llll}
\left(\alpha x_{2} \cdots x_{t}\right) E & O & O & O
\end{array}\right)^{T} \\
H_{f, x} & =\operatorname{EvalFX}\left(f, B_{1}, \cdots, B_{t}, c_{1}, \cdots, c_{t}, x_{1}, \cdots, x_{t}\right) \\
& =\left(\begin{array}{llll}
\left(\alpha x_{2} \cdots x_{t}\right) E & O & O & O
\end{array}\right)^{T}
\end{aligned}
$$

\section{Comparison of these three Techniques}

In this section, we compare our two proposed homomorphic techniques with that used in BGGHNSVV attribute-based encryption scheme (Boneh et al. 2014) in two aspects of efficiency and functionality.

\subsection{Comparison in Efficiency}

The basic homomorphic technique described above is a classical tool in constructions of functional encryption scheme. However, in the process of evaluating multiplication gate, the efficiency is a bottleneck since the procedure cannot be implement-ed in a parallel way. By the construction of the basic homomorphic technique, one has to do the following computation:

$$
G^{-1}\left(-B_{t-1} G^{-1}\left(-B_{t-2} \cdots G^{-1}\left(-B_{1} G^{-1}(\alpha G)\right)\right)\right)
$$

when evaluating the multiplication gate. In above equation, we have to compute the matrix step by step inside and out which is the key problem to result in low efficiency. In order to conquer the weakness of sequential computation, a natural approach is to reduce the number of nests in above computational formula.

In our proposed homomorphic technique I, we need to compute

$$
\alpha G^{-1}\left(-B_{t-1} G^{-1}\left(-B_{t-2} \cdots G^{-1}\left(-B_{1}\right)\right)\right)
$$

Thus, we reduce the number of nests in the computation from $n$ to $n-1$. Hence, our proposed homomorphic technique is more efficient than the basic one.

Though efficiency of the proposed technique $\mathrm{I}$ is improved, the advantage is only one time computat-ion of $G^{-1}$. In our proposed homomorphic technique II, we substantially improve the efficiency since we only need to compute $\left(\alpha x_{2} \cdots x_{t}\right) E$.

\subsection{Comparison in Functionality}

One of vital properties of the basic homomorphic technique which make functional encryption scheme (including attribute-based encryption scheme and predicate encryption scheme) feasible is that during process of computing the matrix $H_{f}$, algorithm $\operatorname{EvalF}(\cdot)$ only takes as input the predicate function $f$ and the public parameters $B_{1}, \cdots, B_{t}$, not including the attribute information $x_{1}, \cdots, x_{t}$. In our proposed homomorphic technique $\mathrm{I}$, this vital property is maintained since $H_{f}$ has nothing to do with the attribute information $x_{1}, \cdots, x_{t}$. However, our proposed homomorphic technique II does not succeed in keeping this property. Therefore, we cannot substitute the basic homomorphic technique with the proposed homomorphic technique II although the latter is more efficient. Nevertheless, replacing with the proposed homomorphic technique I not only ensures the functionality, but also improves the efficiency. 


\section{Conclusions}

In this paper, we analyze the key homomorphic techniques used in the constructions of functional encryption schemes, and point out its weakness in efficiency. In addition, we propose two improved homomorphic techniques and show their advantages and weaknesses through the method of comparison.

\section{ACKNOWLEDGEMENTS}

This work was partially supported by the Doctoral Fund of University of Jinan (Granted No. XBS 1455).

\section{References}

1. Agrawal, Shweta. 2017. Stronger security for reusable garbled circuits, general definitions and attacks. In CRYPTO'17,37th International Cryptology Confer-ence. Springer.

2. Boneh, D., A. Sahai, and B. Waters. 2011. Functional encryption: Definitions and challenges. In TCC'11, 8th Theory of Cryptography Conference. Springer.

3. Boneh, D., C. Gentry, S. Gorbunov, S. Halevi, V. Nikolaenko, G. Segev, V. Vaikuntanathan, and D. Vinayagamurthy. 2014. Fully keyhomomorphic encryption, arithmetic circuit $\mathrm{ABE}$ and compact garbled circuits. In EUROCRYPT'14, 33rd Annual International Conference on the Theory and Applications of Cryptographic Techniques. Springer.

4. Brakerski, Z., R. Tsabary, V. Vaikuntanathan, and H. Wee. Private constrained PRFs (and more) from LWE. In TCC'17, 14th Theory of Cryptography Conference. Springer.

5. Garg, S., C. Gentry, S. Halevi, M. Raykova, A. Sahai, and B. Waters. 2013. Candidate indistinguishability obfuscation and functional encryption for all circuits. In FOCS'13, 54th Annual IEEE Symposium on Foundations of Computer Science. IEEE Computer Society Press.

6. Gentry, Craig. 2009. Fully homomorphic encryption using ideal lattices. In STOC'09, 41th Annual ACM Symposium on the Theory of Computing, ACM Press.

7. Gorbunov, S., V. Vaikuntanathan, and H. Wee. 2013. Attribute-based encryption for circuits. In STOC'13, 45th Annual ACM Symposium on the Theory of Computing, ACM Press.

8. Gorbunov, S., V. Vaikuntanathan, and H. Wee. 2015. Predicate encryption for circuits from LWE. In CRYPTO'15, 35th International Cryptology Conference. Springer. 